\title{
Deciphering the Role of Dipolar Interactions in Magnetic Layered Double Hydroxides
}

\author{
Jose A. Carrasco, ${ }^{\mathrm{a}, \dagger}$ Salvador Cardona-Serra, ${ }^{\mathrm{a}, \mathrm{b}, \dagger}$ Juan M. Clemente-Juan, ${ }^{\mathrm{a}}$ Alejandro \\ Gaita-Ariño, ${ }^{\mathrm{a}, *}$ Gonzalo Abellán, ${ }^{\mathrm{a}, \mathrm{c}, *}$ and Eugenio Coronado ${ }^{\mathrm{a}, *}$ \\ a Instituto de Ciencia Molecular (ICMol), Universidad de Valencia, Catedrático José \\ Beltrán 2, 46980, Paterna, Valencia, Spain. \\ ${ }^{b}$ Trinity College Dublin, College Green, Dublin 2, Ireland. \\ ${ }^{c}$ Department of Chemistry and Pharmacy and Joint Institute of Advanced Materials \\ and Processes (ZMP), University Erlangen-Nürnberg, Henkestr. 42, 91054 Erlangen \\ and Dr.-Mack Str. 81, 90762 Fürth, Germany. \\ ${ }^{\dagger}$ These authors contributed equally to this work.
}

\begin{abstract}
Layered double hydroxides (LDHs) exhibit unparalleled anion exchange properties and the ability to be exfoliated into 2D nanosheets, which retain the properties of the pristine bulk precursor. Still, if one wants to use LDH as a magnetic building block in the design of complex architectures, the role-played by the dipolar magnetic interactions in these layered materials needs to be understood. In this work we synthesized and characterized a five-membered LDH series with basal spacing ranging from 7.5 to $34 \AA$. A detailed investigation combining experimental characterization and theory allows us to conclude that the major factor governing the dipolar interaction between magnetic layers is not the interlayer spacing but the spin correlation size, which in the limit, is related to the lateral dimension of the layer. These results highlight the importance of cation ordering in the magnetic behavior of LDHs, and underpin the differences with homometalliclayered hydroxides.
\end{abstract}




\section{Introduction}

The search of multifunctional materials is one of the most appealing topics in Materials Science. ${ }^{1}$ Among the different strategies, the so-called hybrid approach stands as the most interesting route for the preparation of hybrids by combining organic/molecular and inorganic building blocks. ${ }^{2-4}$ Beyond the coexistence of properties, which is nowadays almost standard, the development of hybrid stimuli-responsive materials has emerged, being a sophisticated tool to control the physical properties of a certain host at will. ${ }^{5,6}$ Among the different available inorganic building blocks, layered metal hydroxides are outstanding candidates due to their chemical versatility and wide range of possible applications. Layered double hydroxides (LDH) are a special class of anionic clays consisting of positively charged brucite-type inorganic layers interleaved with anions. ${ }^{7,8}$ They can be formulated as $\left[\mathrm{M}^{\mathrm{II}}{ }_{1-\mathrm{x}} \mathrm{M}^{\mathrm{III}}{ }_{\mathrm{x}}(\mathrm{OH})_{2}\right]^{\mathrm{x}+}\left[\mathrm{A}^{\mathrm{n}-}\right]_{\mathrm{x} / \mathrm{n}} \cdot m \mathrm{H}_{2} \mathrm{O}$ $\left(\mathrm{A}^{\mathrm{n}-}=\right.$ organic or inorganic anions). They exhibit unparalleled anion exchange properties and a wide chemical versatility. One of theit most interesting properties is the ability to be exfoliated into 2D nanosheets, which can be used as macromolecular building blocks in the design of more complex architectures, as they retain the properties of the pristine bulk precursor. ${ }^{9}$ LDHs can be synthesized using first row paramagnetic transition metal ions that, in combination with the ability to confine guest molecules, originate a plethora of magnetic behaviours, however dipolar effects have never been studied in detail. In brief, the magnetic properties of LDH and their hybrids are determined by two main contributions: the in-plane superexchange mechanism mediated by $-\mathrm{OH}$ bridges on the one hand, and the inter-plane weaker dipolar interactions. ${ }^{10,11}$ While the replacement of the diamagnetic $\mathrm{Al}(\mathrm{III})$ centres by paramagnetic cations provide a rich magnetic behaviour due to the competition between ferro- and antiferromagnetic interactions, the role of dipolar interactions is much less understood. ${ }^{12}$ Indeed, we recently reported that when a photo-active molecule is rigidly connected to two adjacent LDH layers, the magnetic properties change strongly as a consequence of the distortion induced within the layers when the hybrid material is irradiated. Still, one expects that during this process the dipolar interactions remain unchanged since the interlayer distances do not change. ${ }^{10}$ In contrast, when these linkers are only connected by one side to the LDH layers, these layers are not distorted and, as a consequence, there is only a slight change in the magnetic properties exclusively affected by dipolar interactions. ${ }^{11}$ While interlayer distances could be a straightforward path to control magnetic properties in layered materials, the factors governing dipolar interactions in LDH 
remain unclear for the scientific community despite some recent remarkably efforts. ${ }^{13,14}$ Such an understanding and its relationship with the structural features of the layered material is a matter of utmost importance for designing stimuli-responsive systems. To fill this gap, herein we propose a combined experimental and theoretical study using as model system a highly crystalline CoAl-LDH with well-defined hexagonal shapes intercalated with a series of $n$-alkyl sulfates of different length (from 2 to 18). This allows us to modify the basal space (BS) in a wide range (from ca. 7.6 to $33.8 \AA$ ), while keeping the most simple magnetic scenario for LDHs, consisting of only one magnetic element (i.e. Co(II)). Thus, a systematic study of the influence of the dipolar interactions on the magnetism of LDHs can be performed, shedding light on their complex magnetic behaviour and paving the way for tuning its magnetic properties at will.

\section{Synthesis of CoAl-LDHs}

Chemicals: $\mathrm{CoCl}_{2} \cdot 6 \mathrm{H}_{2} \mathrm{O}, \mathrm{AlCl}_{3} \cdot 6 \mathrm{H}_{2} \mathrm{O}$, urea $\left(\mathrm{CH}_{4} \mathrm{~N}_{2} \mathrm{O}\right), \mathrm{NaNO}_{3}$, sodium ethyl sulfate $\left(\mathrm{C}_{2} \mathrm{H}_{5} \mathrm{NaO}_{4} \mathrm{~S}\right)$, sodium octyl sulfate $\left(\mathrm{C}_{8} \mathrm{H}_{17} \mathrm{NaO}_{4} \mathrm{~S}\right)$, sodium dodecyl sulfate $\left(\mathrm{C}_{12} \mathrm{H}_{25} \mathrm{NaO}_{4} \mathrm{~S}\right)$ and sodium octadecyl sulfate $\left(\mathrm{C}_{18} \mathrm{H}_{37} \mathrm{NaO}_{4} \mathrm{~S}\right)$ were purchased from Sigma-Aldrich. Ethanol absolute was purchased from Panreac. All chemicals were used as received. Ultrapure water was obtained from a Millipore Milli-Q equipment.

Synthesis of CoAl-CO $\mathrm{CO}_{3}$ LDH: Following the method described by Liu et al ${ }^{15}$

In a typical procedure, $0.02 \mathrm{M}$ of $\mathrm{CoCl}_{2} \cdot 6 \mathrm{H}_{2} \mathrm{O}, 0.01 \mathrm{M}$ of $\mathrm{AlCl}_{3} \cdot 6 \mathrm{H}_{2} \mathrm{O}$ and $0.07 \mathrm{M}$ of urea were dissolved in $500 \mathrm{~mL}$ of Milli-Q water. The mixture was set under $\mathrm{Ar}$ atmosphere and magnetic stirring for $48 \mathrm{~h}$ at the refluxing temperature of $97^{\circ} \mathrm{C}$. Then, the mixture was filtered, washed with Milli-Q water and EtOH and dried at room temperature. The resulting pink pale powder was grinded and collected as the final product.

Synthesis of CoAl-NO $\mathrm{CDH}_{3}$ : $1.5 \mathrm{M}$ of $\mathrm{NaNO}_{3}$ and $0.005 \mathrm{M}$ of $\mathrm{HNO}_{3}$ were dissolved in $500 \mathrm{~mL}$ of Milli-Q water. Then, $500 \mathrm{mg}$ of $\mathrm{CoAl}-\mathrm{CO}_{3} \mathrm{LDH}$ were added to the mixture and the reaction was set under Ar atmosphere and magnetic stirring for $96 \mathrm{~h}$. Finally, the mixture was filtered, washed with Milli-Q water and EtOH and dried in a vacuum. The resulting pink pale powder was grinded and collected as the final product.

Synthesis of CoAl-ES and CoAl-OS LDHs: $90 \mathrm{~mL}$ of Milli-Q water were placed in a two-neck flask and set under Ar atmosphere and magnetic stirring at $50{ }^{\circ} \mathrm{C}$. After 30 min, $0.03 \mathrm{M}$ of Sodium Ethyl Sulfate or Sodium Octyl Sulfate (SES or SOS, 
respectively) was added to the solution, followed by the addition of $200 \mathrm{mg}$ of CoAl$\mathrm{NO}_{3} \mathrm{LDH}$ as soon as the SES/SOS was completely dissolved. The reaction was kept for 48 h. Finally, the mixture was filtered, washed with Milli-Q water and EtOH and dried in a vacuum. The resulting pink pale powder was grinded and collected as the final product.

Synthesis of CoAl-DS LDH: $50 \mathrm{~mL}$ of Milli-Q water was placed in a two-neck flask and set under Ar atmosphere and magnetic stirring at $50{ }^{\circ} \mathrm{C}$. After $30 \mathrm{~min}, 0.2 \mathrm{M}$ of Sodium Dodecyl Sulfate (SDS) was added to the solution, followed by the addition of $200 \mathrm{mg}$ of $\mathrm{CoAl}-\mathrm{NO}_{3} \mathrm{LDH}$ as soon as the SDS was completely dissolved. The reaction was kept for 12 h. Finally, the mixture was filtered, washed with Milli-Q water and EtOH and dried in a vacuum. The resulting pink pale powder was grinded and collected as the final product.

Synthesis of CoAl-ODS LDH: $50 \mathrm{~mL}$ of Milli-Q water was placed in a two-neck flask and set under $\mathrm{Ar}$ atmosphere and magnetic stirring at $65{ }^{\circ} \mathrm{C}$. After $30 \mathrm{~min}, 0.01 \mathrm{M}$ of Octadecyl Sodium Sulfate (ODSS) was added to the solution, followed by the addition of $200 \mathrm{mg}$ of $\mathrm{CoAl}-\mathrm{NO}_{3} \mathrm{LDH}$ as soon as the OSS was completely dissolved. The temperature was set at $50{ }^{\circ} \mathrm{C}$. The reaction was kept for $12 \mathrm{~h}$. Finally, the mixture was filtered, washed with Milli-Q water and EtOH and dried in a vacuum. The resulting pink pale powder was grinded and collected as the final product.

\section{Physical Characterization.}

X-ray powder diffraction (XRPD) patterns were obtained with a Bruker D8 Advance A25 using the copper radiation $(\mathrm{Cu}-\mathrm{K} \alpha=1.54178 \AA)$ in the $2-40^{\circ}$ region. Field emission scanning electron microscopy (FESEM) studies were carried out on a Hitachi S-4800 microscope at an accelerating voltage of $20 \mathrm{kV}$ and 60 seconds of $\mathrm{Au} / \mathrm{Pd}$ metallization of the samples. ATR Infrared spectra were collected in an Agilent Cary 630 FTIR spectrometer in the $4000-650 \mathrm{~cm}^{-1}$ range in absence of $\mathrm{KBr}$ pellets. Thermogravimetric analysis (TGA) of all samples were carried out in a Mettler Toledo TGA/SDTA 851 apparatus in the $25-800{ }^{\circ} \mathrm{C}$ temperature range at $10{ }^{\circ} \mathrm{C} \cdot \mathrm{min}^{-1}$ scan rate and an air flow of $30 \mathrm{~mL} \cdot \mathrm{min}^{-1}$. Carbon, nitrogen, hydrogen and sulphur contents were determined by microanalytical procedures by using a LECO CHNS. Magnetic data were collected over the bulk material with a Quantum Design superconducting quantum interference device (SQUID) MPMS-XL-5. The magnetic susceptibility of the samples was corrected from the diamagnetic contributions of their atomic constituents as deduced from Pascal's constant tables and the sample holder. The dc data were obtained 
under an external applied filed of 100 or 1000 Oe in the $2-300 \mathrm{~K}$ temperature range. Magnetization studies were performed between -5 and $+5 \mathrm{~T}$ at a constant temperature of $2 \mathrm{~K}$. The ac data were collected under an applied field of 3.95 Oe at 997, 333, 110, 10 and $1 \mathrm{~Hz}$.

\section{Results and discussion}

In order to have a material with a high crystallinity and well-defined morphology we selected the CoAl-LDH prepared by a modified homogeneous precipitation method using urea as the ammonia-releasing reagent (ARR). ${ }^{11}$ This LDH exhibits an excellent anion exchange capability, being possible to obtain the $\mathrm{NO}_{3}{ }^{-}$form under mild acid-salt treatment. By subsequent anion exchange of the nitrate form with four different $n$-alkylsulfate derivatives, we obtained a family of materials with an increasing number of carbon atoms: 2 for the ethyl (-ES), 8 for the octyl (-OS), 12 for the dodecyl (-DS) and 18 for the octadecyl (-ODS) form. ${ }^{11,15}$ It is worth to highlight the importance of the synthetic conditions for each sample, since the synthesis for every surfactantintercalated material requires some specific conditions. Moreover, to the best of our knowledge, the -ES and -ODS CoAl-LDH derivatives have never been reported so far. The complete characterization of the $\mathrm{CoAl}-\mathrm{NO}_{3}$ precursor can be encountered in supporting information SI 10. X-ray powder diffraction (XRPD) patterns of the studied CoAl-LDH family exhibit the characteristic basal reflections observed in these class of materials, showing an excellent crystallinity for all of them and a concomitant shift towards lower 2-Theta values as the length of the intercalated anion is increased. ${ }^{16}$ The values of the observed BS varied from. $7.6 \AA$ for the $\mathrm{CoAl}-\mathrm{CO}_{3}$ sample to $33.8 \AA$ for CoAl-ODS sample (Fig. 1A and Table 1) ${ }^{11,17}$ It is worth to mention that for large surfactants like DS or ODS there is an enhancement of the crystallinity, leading to better-defined crystallographic peaks, showing up to 10 basal reflections. ${ }^{18-20}$ This effect is attributed to the stabilization during the synthesis of the LDH nanoplatelets thanks to the electrostatic interactions between the cationic sheets and the anionic polar head of the surfactant, whereas the hydrocarbonated apolar chain creates a micelle system in the reaction media. ${ }^{21,22}$ The graphic correlation between basal space and 2-Theta values can be found in Fig. 1B. 
Table 1. Compositional information of the studied CoAl-LDH family. The alkyl-sufates used are: ethyl sulfate (ES), octyl sulfate (OS), dodecyl sulfate (DS) and octadecyl sulfate (ODS).

\begin{tabular}{ccccc}
\hline Sample & $\begin{array}{c}\text { Carbon } \\
\text { Number }\end{array}$ & $\begin{array}{c}\text { Basal } \\
\text { space }(\AA)\end{array}$ & Molecular formula $^{\text {a }}$ & $\begin{array}{c}\text { Ratio } \\
\text { Co:Al }\end{array}$ \\
\hline CoAl-CO & 1 & 7,59 & {$\left[\mathrm{Co}_{0,67}{ }^{2+} \mathrm{Al}_{0,33}{ }^{3+}(\mathrm{OH})_{2}\right]^{0,34+}\left(\mathrm{CO}_{3}{ }^{2-}\right)_{0,17} \cdot 0.33 \mathrm{H}_{2} \mathrm{O}$} & 2,03 \\
CoAl-ES & 2 & 14,96 & {$\left[\mathrm{Co}_{0,67}{ }^{2+} \mathrm{Al}_{0,33}{ }^{3+}(\mathrm{OH})_{2}\right]^{0,34+}\left(\mathrm{ES}^{-}\right)_{0,33} \cdot 1.51 \mathrm{H}_{2} \mathrm{O}$} & 2,03 \\
CoAl-OS & 8 & 21,76 & {$\left[\mathrm{Co}_{0,66}{ }^{2+} \mathrm{Al}_{0,34^{3+}}(\mathrm{OH})_{2}\right]^{0,34+}\left(\mathrm{OS}^{-}\right)_{0,34} \cdot 1.49 \mathrm{H}_{2} \mathrm{O}$} & 1,94 \\
CoAl-DS & 12 & 26,68 & {$\left[\mathrm{Co}_{0,66}{ }^{2+} \mathrm{Al}_{0,34}{ }^{3+}(\mathrm{OH})_{2}\right]^{0,34+}\left(\mathrm{DS}^{-}\right)_{0,34} \cdot 1.70 \mathrm{H}_{2} \mathrm{O}$} & 1,94 \\
CoAl-ODS & 18 & 33,84 & {$\left[\mathrm{Co}_{0,67}{ }^{2+} \mathrm{Al}_{0,33}{ }^{3+}(\mathrm{OH})_{2}\right]^{0,34+}\left(\mathrm{ODS}^{-}\right)_{0,33} \cdot 1.49 \mathrm{H}_{2} \mathrm{O}$} & 2,03 \\
\hline
\end{tabular}

${ }^{a}$ Molecular formula was estimated from the EDAX microanalysis alongside with TGA and elemental analysis results.

FTIR spectra (Fig. 1C) were collected to provide further information regarding the anionic intercalation. Among the different vibrational bands appearing in the spectra, it is noticeable to highlight the $\mathrm{C}-\mathrm{H}$ stretching doublet at $c a .2917$ and $2845 \mathrm{~cm}^{-1}$. This band is related with the presence of the surfactant, ${ }^{23}$ and gains intensity as long as the length of the molecule is increased. Concretely, methylene scissoring vibrational mode $\left(2845 \mathrm{~cm}^{-1}\right)$ and sulfate asymmetric and symmetric stretching bands (1184 and $1050 \mathrm{~cm}^{-}$ ${ }^{1}$ ) can be clearly detected in the surfactant-intercalated compounds. ${ }^{23}$ According to the model stated by Lagaly, we can expect a bilayer arrangement of the surfactants within the LDH sheets. ${ }^{24}$

TGA and elemental analysis were carried out to gain further evidence of the successfully exchange procedures. Fig. 1D depicts the TGA patterns of the whole CoAlLDH family (see SI 1 for a single focused TGA/DTA plot on each sample). These TGA can be splitted out in two main segments including several processes. ${ }^{25-27}$ First of all, a low-temperature weight loss between $25-220^{\circ} \mathrm{C}$ related with the removal of hydrated and intercalated (physisorbed) water molecules, followed by a second weight loss in the $220-600{ }^{\circ} \mathrm{C}$ related with the evaporation of the interlamellar (chemisorbed) water molecules and the dehydroxilation of the layers, as well as the decomposition of the organic molecule. ${ }^{26,28}$ Interestingly, an increase in the final weight loss can be observed 
after the exchange of larger surfactant molecules, in accordance with the theoretical molecular formula. EDAX microanalysis was used to confirm that the experimental ratio between $\mathrm{Co}$ and $\mathrm{Al}$ atoms ( $\mathrm{Co} / \mathrm{Al}$ ratio of 2). The structural information of all samples is summarized in Table 1. Comparison between calculated and experimental elemental analysis values can be found in SI 2, exhibiting excellent agreement.
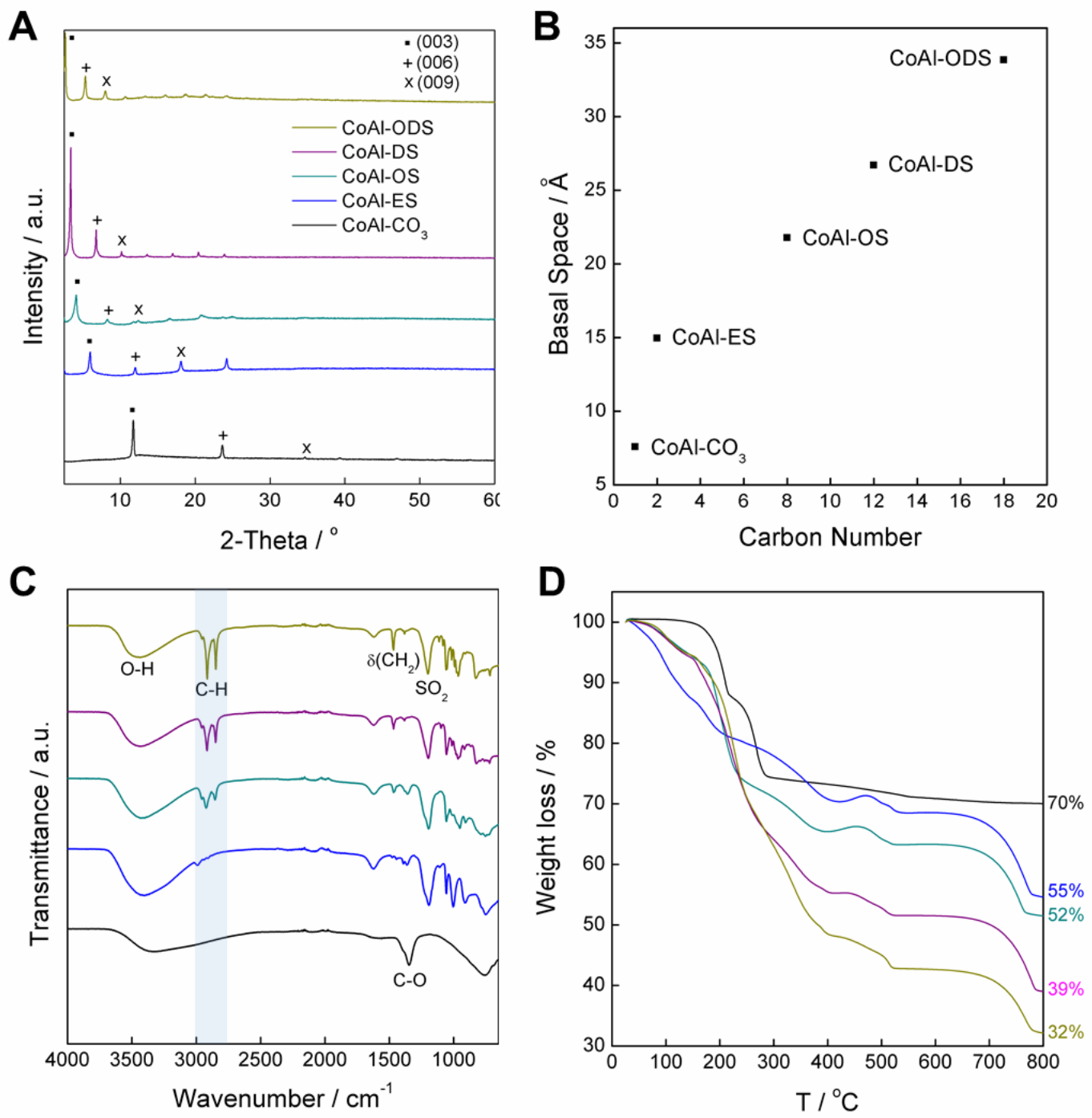

Fig. 1. (A) XRPD patterns of the CoAl-LDHs in the $2-60^{\circ}$ region. (B) Basal space versus carbon number. (C) FTIR spectra of the CoAl-LDH family in absence of $\mathrm{KBr}$ pellet. Main vibrational bands are pointed out and alkyl bands related with the surfactants are highlighted. (D) TGA in air for the synthesized samples, highlighting the total weight loss according to the anion intercalation. 
In order to study the morphology of these materials and the influence of the successive anion exchange process in the shape and size of the crystals we have used fieldemission scanning electron microscopy (FESEM). ${ }^{29,30}$ Fig. 2, reveals that their welldefined hexagonal shape is maintained independent of the selected interlamellar anion. SI 3 and SI 4 depict low-magnification FESEM images as well as a mapping analysis of $\mathrm{CoAl}-\mathrm{CO}_{3}$, respectively, to check the homogeneous distribution of $\mathrm{Co}$ and $\mathrm{Al}$ atoms throughout the layers.

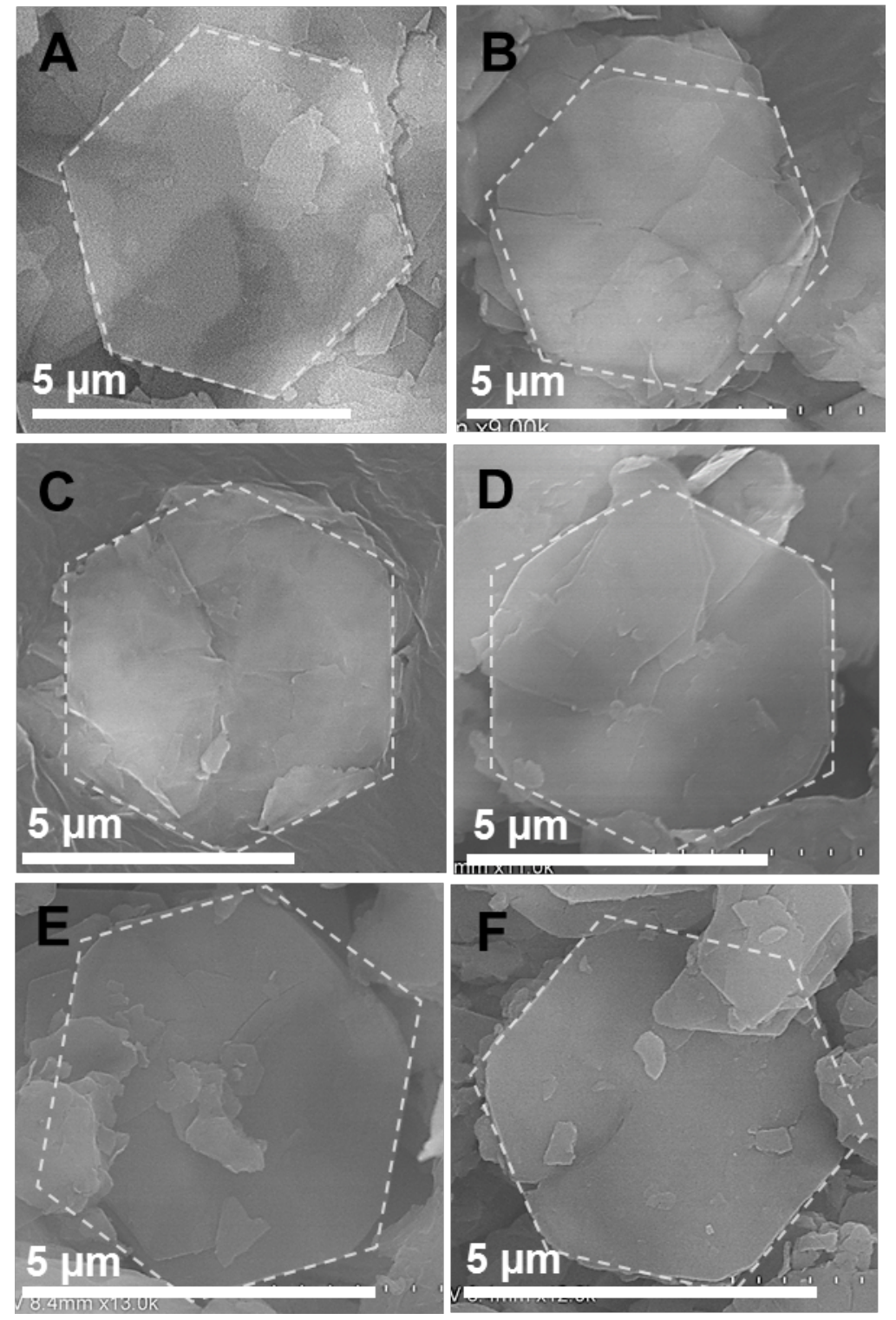

Fig. 2. FESEM images of the CoAl-LDH family, highlighting that the hexagonal morphology is retained after the anionic exchange reaction. (A) $\mathrm{CoAl}_{-} \mathrm{CO}_{3}$, (B) CoAl$\mathrm{NO}_{3}$, (C) CoAl-ES, (D) CoAl-OS, (E) CoAl-DS and (F) CoAl-ODS. 
Average lateral sizes and thicknesses values for all samples were also compared in Fig. 3A and 3B, respectively. Whereas the lateral dimensions of the platelets remain constant in every case, an increase in the platelet thickness is observed as long as the carbon number of the sample is increased, from a minimum of $c a .120 \mathrm{~nm}$ for 1 carbon atom to a maximum of $c a$. $250 \mathrm{~nm}$ for the octadecyl sample. This effect is clearly related with the continuous expanding of the LDH system with the inclusion of larger anions within their layered cationic sheets, confirming the topochemical reaction of intercalation, as previously reported by Ogawa et al. ${ }^{11,31}$ Fig. 3C depicts the chemical structure of the different surfactant molecules here studied. 
A

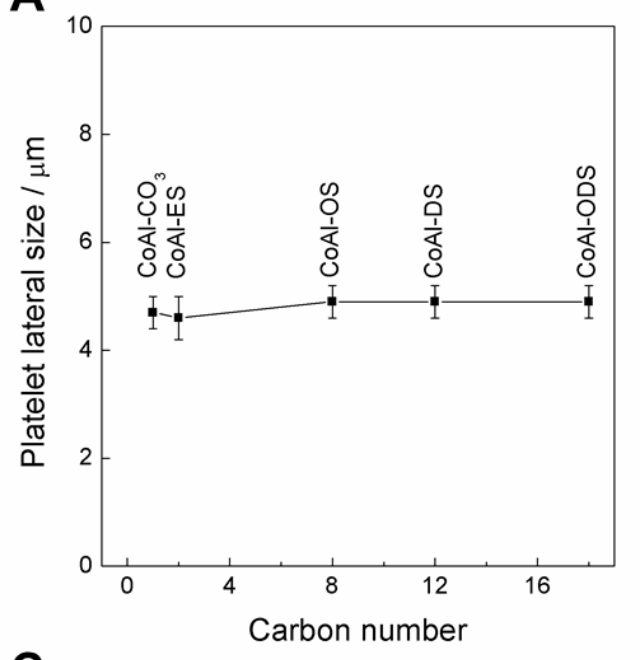

B

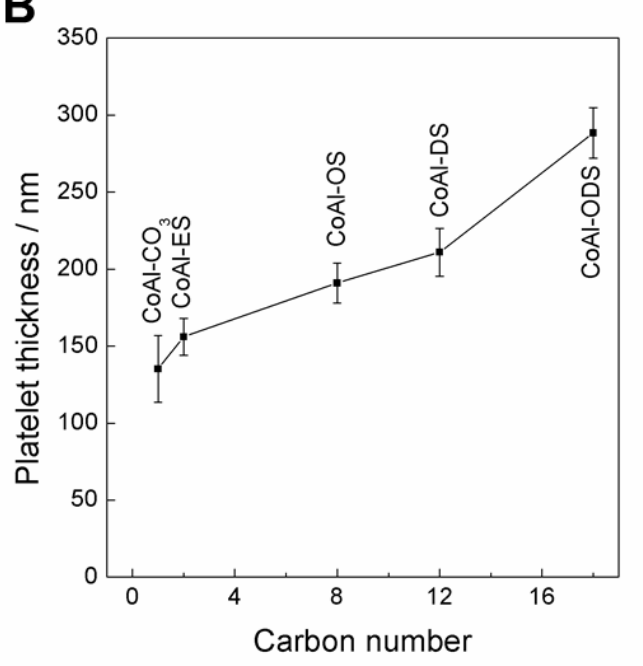

C

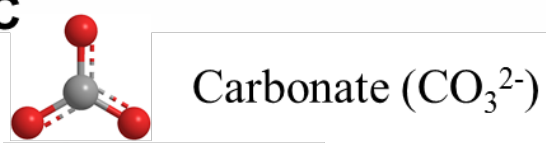

Ethyl sulfate (ES)

al octyl sulfate $\left(\mathrm{OS}^{-}\right)$

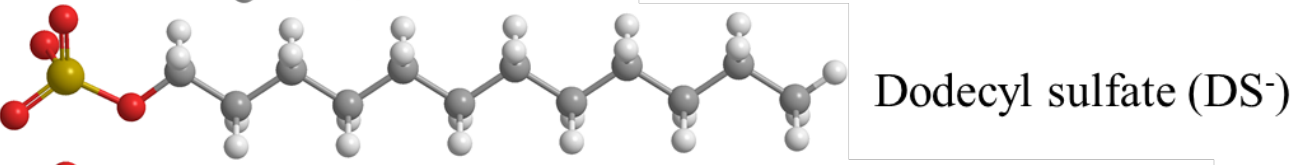

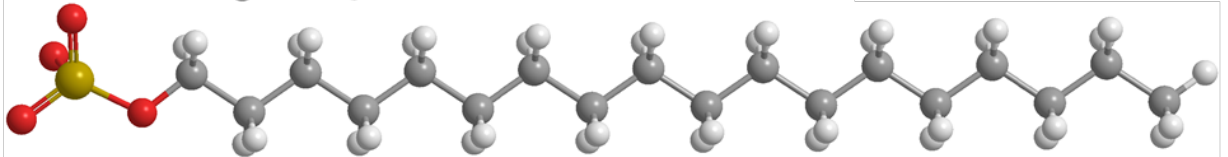

Octadecyl sulfate (ODS') 
Fig. 3. (A) Mean platelet lateral size with its standard deviation and (B) approximated platelet thickness values for every CoAl-LDH sample determined by FE-SEM. Individual histograms for each sample highlighting their lateral size distribution can be found in SI 5. (C) Surfactant molecules used as separation bridges.

An exhaustive magnetic study has been made on these CoAl-LDH intercalated compounds. The overall magnetism in this class of materials is controlled by two main contributions $^{8}$ namely magnetic superexchange interactions, which occur between neighboring metallic centers mediated by hydroxyl bridges $(-\mathrm{OH})$ within each single layer (intralayer interaction), and dipolar magnetic interactions that take place between different layers (interlayer interaction). Because of the strong radial dependence of dipole-dipole interaction, one could expect the dipolar interactions to be intrinsically dependent of the distance between the cationic sheets in the global system; therefore, an increase in the spacing between the layers should affect the overall magnetism of the material. However, several parameters like particle size and morphology, flexibility of the layers or cation clustering affect the magnetic behavior of LDHs as well. According to the reported studies, Al-containing-, NiMn- and NiCr-LDHs exhibit AF dipolar interactions, whereas $\mathrm{NiFe}-\mathrm{LDHs}$ reveal dipolar interactions of ferromagnetic character. ${ }^{8}$ This is in sharp contrast to the results reported for homometallic-layered hydroxides, in which the hydroxide layers are covalently connected to the interlamellar anions. $^{32-34}$ This strong bond seems to exert an important role in their interlayer magnetic interactions. In fact, for shorter interlayer distances of less than $1 \mathrm{~nm}$, throughbond AF interactions appear, promoting metamagnetic behaviour. On the other hand, for higher interlayer distances (> $1 \mathrm{~nm}$ ) dipolar $\mathrm{F}$ through-space interactions predominate, with a significant distance-dependence between individual spins, likely proportional to $B S^{-3}{ }^{32,35-37,34}$ Concerning LDHs, only dipolar interactions should appear, due to the noncovalent connection between the layers and the anions. In this sense, related precedents reporting on the influence of $B S$ on the overall magnetism exhibited dispare results. Firstly, a family of sub-nanometric hybrid co-precipitated NiFe-LDHs, suggesting F dipolar interactions, in which the temperature for the spontaneous magnetization $\left(T_{M}\right)$ and the coercivity decrease proportionally to $B S^{-3} \cdot{ }^{14}$ Still, the nature of the interlamellar anion could promote some divergences to this tendency, as observed for mononuclear macrocyclic-intercalated LDHs. ${ }^{38-40}$ Secondly, a family of micrometric n-alkylsulfonates intercalated CoNi-LDHs with interlayer distances up to $22.41 \AA$, showing a variation in 
the coercive field with the basal space with no clear trend, and an increase for the maximum interlayer distances.

Herein we will try to unveil how the system is affected by a continuous displacement of the layers in a unprecedented wide range due to the anion exchange of successive larger molecules (in the form of surfactants), keeping intact the metallic composition, lateral dimensions and morphology of the crystals.

Table 2 summarizes all the experimental magnetic parameters calculated for the CoAlLDH family. For the sake of clarity, only the magnetic graphs for the sample exhibiting the maximum BS value, CoAl-ODS, are included in the main text (Fig. 4). The complete magnetic data of each sample can be found in SI $6\left(\mathrm{CoAl}-\mathrm{CO}_{3}, \mathrm{CoAl}-\mathrm{ES}\right.$, CoAl-OS and CoAl-DS) and SI 7 (CoAl-NO $\mathrm{NO}_{3}$. 
A
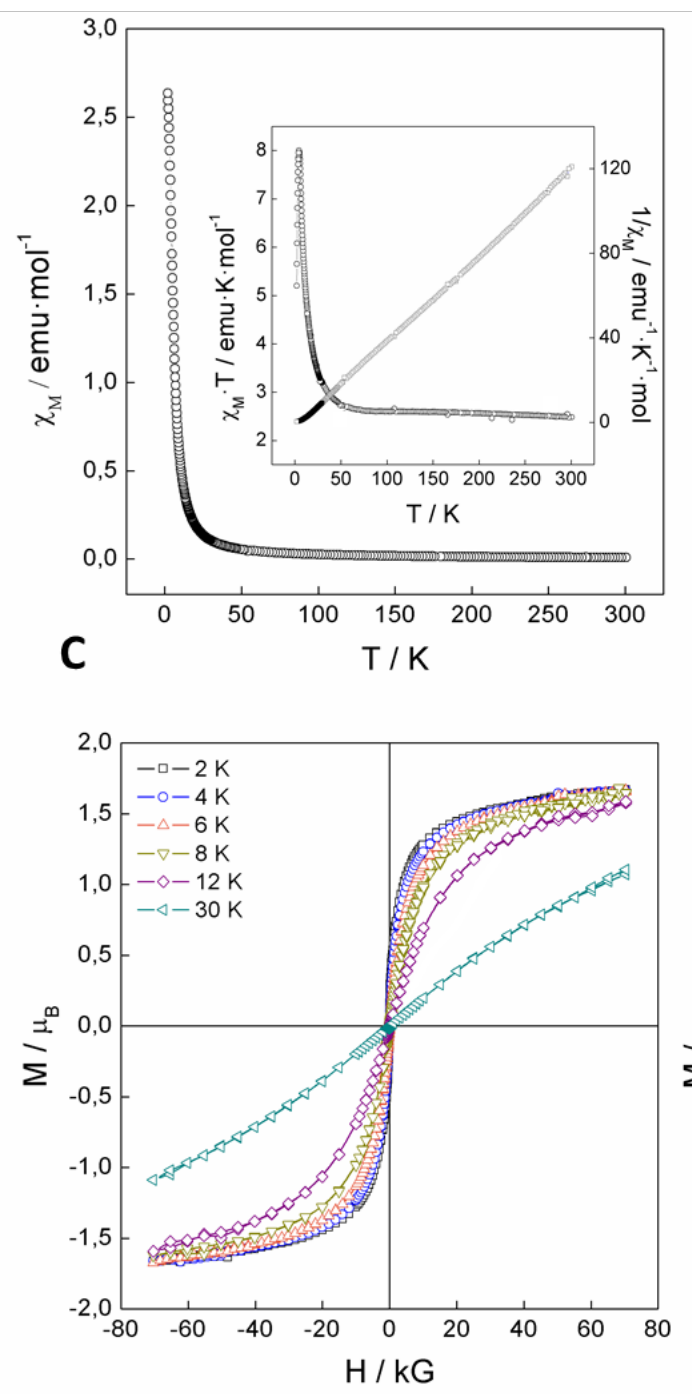

B
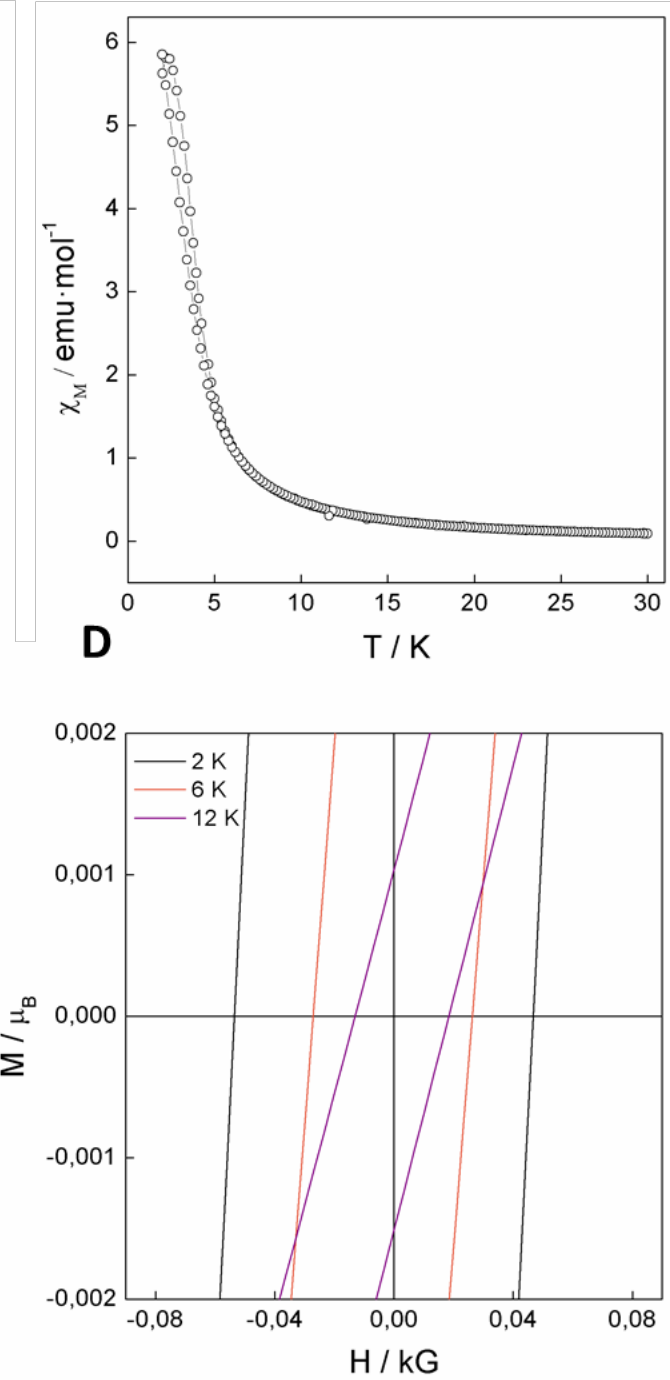

Fig. 4. Magnetic properties of the CoAl-ODS sample: (A) $\chi_{M}$ vs. $T$ plot. The inset represents the temperature dependence of the $\mathrm{x} \cdot \mathrm{T}$ product and the fitting of the data to a Curie-Weiss law. (B) FC/ZFC plot. (C) Hysteresis at different temperatures. (D) The inset shows the low field region for 2, 6 and $12 \mathrm{~K}$.

Table 2. Main magnetic data and parameters for the magnetic CoAl-LDHs. ${ }^{a}$

\begin{tabular}{|c|c|c|c|c|c|c|c|c|}
\hline Sample & $\begin{array}{c}\mathrm{X} \cdot \mathrm{T}_{\mathrm{RT}} \\
\left(\mathrm{emu} \cdot \mathrm{K} \cdot \mathrm{mol}^{-1}\right)\end{array}$ & $\begin{array}{c}\mathrm{C}_{\text {so }} \\
\left(\mathrm{emu} \cdot \mathrm{K} \cdot \mathrm{mol}^{-1}\right)\end{array}$ & $\begin{array}{c}\mathrm{C} \\
\left(\mathrm{emu} \cdot \mathrm{K}^{\prime} \cdot \mathrm{mol}^{-1}\right)\end{array}$ & $\theta(\mathbf{K})$ & $\begin{array}{r}\mathbf{T}_{\mathbf{B}} \\
(\mathbf{K})\end{array}$ & $\begin{array}{l}\mathbf{T}_{\mathbf{M}} \\
(\mathbf{K})\end{array}$ & $\begin{array}{l}M_{S} \\
\left(\mu_{B}\right)\end{array}$ & $\begin{array}{c}\mathbf{H}_{\mathrm{c}} \\
(\mathrm{Oe})\end{array}$ \\
\hline $\mathrm{CoAl}-\mathrm{CO}_{3}$ & 2,00 & 1,26 & 2,02 & $-0,04$ & 2,81 & $\begin{array}{c}4,8 \\
0\end{array}$ & 1,26 & 13,66 \\
\hline CoAl-ES & 2,48 & 1,26 & 2,49 & 4,04 & 2,35 & $\begin{array}{c}5,4 \\
1\end{array}$ & 1,56 & 20 \\
\hline CoAl-OS & 2,48 & 1,24 & 2,49 & $-0,45$ & 2,18 & 5,0 & 1,52 & 30 \\
\hline
\end{tabular}




\begin{tabular}{ccccccccc} 
& & \multicolumn{7}{c}{0} \\
CoAl-DS & 2,50 & 1,24 & 2,51 & 3,13 & 2,29 & 4,8 & 1,52 & 40 \\
CoAl-ODS & 2,48 & 1,26 & 2,47 & $-5,85$ & 5,60 & 4,8 & 1,66 & 48 \\
\hline
\end{tabular}

${ }^{a}$ From left to right: $\chi \cdot T_{\mathrm{RT}}$ value at room temperature; expected spin-only value of the Curie constant $\left(\mathrm{C}_{\mathrm{so}}\right)$; experimental Curie constant $(\mathrm{C})$; Weiss constant $(\theta)$; temperature of the divergence of the ZFC and FC magnetic susceptibility $\left(\mathrm{T}_{\mathrm{B}}\right)$; temperature for the onset of spontaneous magnetization extracted from the $\chi^{\text {” }}{ }_{\mathrm{M}}$ plot $\left(\mathrm{T}_{\mathrm{M}}\right)$; measured hysteresis temperature $\left(\mathrm{T}_{\mathrm{Hys}}\right)$; saturation magnetization $\left(\mathrm{M}_{\mathrm{S}}\right)$; coercive field $\left(\mathrm{H}_{\mathrm{Coer}}\right)$. $\mathrm{S}\left(\mathrm{Co}^{2+}\right)=3 / 2, \mathrm{~S}\left(\mathrm{Al}^{3+}\right)=0$.

The DC susceptibility measurements (Fig. 4A) $\left(\chi_{M}\right)$ for the CoAl-ODS sample exhibit a continuous increase upon cooling down, which becomes very sharp at low T (below 20 $\mathrm{K}$ ) reaching a maximum value of $2.63 \mathrm{emu} \cdot \mathrm{mol}^{-1}$ at $2 \mathrm{~K}$. In addition, the $\chi_{M} \cdot T$ product also increases exhibiting a sharp peak in the low $\mathrm{T}$ range. Thus, the thermal variation of $\chi_{\mathrm{M}} \cdot \mathrm{T}$ slightly increases from a value of $2.48 \mathrm{emu} \cdot \mathrm{K} \cdot \mathrm{mol}^{-1}$ at room temperature to a value of $2.60 \mathrm{emu} \cdot \mathrm{K} \cdot \mathrm{mol}^{-1}$ at $70 \mathrm{~K}$, followed by a sharp increase up to $8.03 \mathrm{emu} \cdot \mathrm{K}^{\prime} \mathrm{mol}^{-1}$ at 4.6 $\mathrm{K}$ and a rapid decrease to $5.2 \mathrm{emu} \cdot \mathrm{K} \cdot \mathrm{mol}^{-1}$ at $2 \mathrm{~K}$. The inset of Fig. $4 \mathbf{A}$ also presents the fitting of the inverse of the $\chi_{M}$ to a Curie-Weiss law above $50 \mathrm{~K}$, resulting in a Curie constant (C) of $2.47 \mathrm{emu} \cdot \mathrm{K} \cdot \mathrm{mol}^{-1}$, higher than that expected for a spin only value of a magnetically diluted combination of $\mathrm{Co}^{2+}(\mathrm{S}=3 / 2)$ and $\mathrm{Al}^{3+}(\mathrm{S}=0)$, and a small and negative value of the Weiss constant $(\theta)$. All these results are consistent with the presence of dominant ferromagnetic interactions within the layers. In the present case, the weak negative value of $\theta$, does not seem to be due to the predominance of antiferromagnetic interactions but to the orbital contribution coming from octahedral Co(II).

In different samples one observes no apparent correlation between the $\chi_{\mathrm{m}} \cdot \mathrm{T}$ vs $\mathrm{T}$ plots and the interlayer distance (see SI 6). It is worth noting that, according to Table 2, $\theta$ does not show a linear trend with the increase of the anion allocated within the layers. Moreover, in the case of the sample with the smallest interlamellar space $\left(\mathrm{CoAl}-\mathrm{CO}_{3}\right)$, we also measured the field dependence of $\chi$ vs $T$ between 100 and $5000 \mathrm{G}$ to rule out any possible metamagnetic behavior (see SI 6.1.1). This result clearly differentiates the LDH behavior to that of the monometallic hydroxides as pointed out previously. ${ }^{16,34}$ 
For the CoAl-ODS sample field cooled and zero field cooled (FC/ZFC) data (Fig. 4B) allows us to extract a value for blocking temperature of $5.60 \mathrm{~K}$, which is the highest value of the family. Hysteresis loop was recorded at different temperatures (Fig 4C), depicting spontaneous magnetization at low temperatures with a value of 48 Oe at $2 \mathrm{~K}$ (Fig 4D), which is also the highest value of the family. Interestingly, a clear trend was observed for the values of coercive field $\left(\mathrm{H}_{\mathrm{C}}\right)$, which increase with the basal space. The relative low values (ca. $10-50$ Oe) are indicative of soft magnets, and are far from other LDHs with hard magnetic behavior ( $>1000$ Oe as $\mathrm{H}_{\mathrm{C}}$ ). ${ }^{12,41}$ In addition, we have studied the influence of the temperature on the presence of hysteretic behavior in the magnetization vs. field plot for the CoAl-ODS sample (Fig. 4C and 4D). Below the blocking temperature $\left(T_{B}=5.6 \mathrm{~K}\right)$ the curves can be superimposed exhibiting the same coercive field. Above this value a progressive decrease in the coercive field and the remnant magnetization can be measured, indicative of superparamagnetic-like behavior. ${ }^{41-43}$ In sum, experimentally we have a somewhat puzzling situation in which a systematically increase in the interlayer spacing does not afford a clear dependence of the magnetic behavior, except for the coercive field.

To complement this study from the theoretical point of view, we have proposed two approaches to understand the correlation (if any) between the static magnetic behaviour and the interlayer distance: i) a semiempirical fitting of the experimental magnetic susceptibility with an anisotropic spin Hamiltonian including an effective interlayer coupling parameter between minimal hexagonal networks, and ii) a purely theoretical study which considers the magnetic dipolar interaction along a range of interlayer distances and magnetic domain sizes. We make no attempt to reproduce the dynamic magnetic behavior. Even so, because of the enormous magnetic complexity of these systems and the limited experimental data available, theoretical models are necessarily oversimplifications which may have limited validity. Thus, we include here only a summary and collection of the most robust conclusions, with all details being available in SI sections SI 8 to SI 13.

Satisfactory fittings of the thermal dependences of the magnetic susceptibility were obtained using the previously mentioned model (see SI 8). A minimal six-member network was used to model each layer since larger models were either inadequate for symmetry reasons or unsolvable for computational reasons (see SI 9). The Hamiltonian considers anisotropic exchange between effective spin Kramers doublets (allowing 
$\mathrm{Jz} \neq \mathrm{Jxy}$ ), an effective interlayer coupling and a Zeeman term, also allowing gz $\neq$ gxy (see SI 10 for the complete fitting set and SI 11 for the parameters obtained on each case). Fittings with a smaller number of degrees of freedom were attempted but were not successful (See SI 12). While overparametrization impedes extracting fine details of these fits, a few main trends seem stable among different fitting attempts, namely (i) the Co(II) ions, which are here in a very axially distorted environment, behave as Ising spins, (ii) $\mathrm{Co}(\mathrm{II})-\mathrm{O}-\mathrm{Co}(\mathrm{II})$ superexchange interactions are in the usual range reported for oxo-bridged complexes of similar geometries, and (iii) the interlayer exchange interaction is two orders of magnitude weaker compared with the superexchange interaction.

The purely dipolar model was explored in order to obtain some general understanding about the importance of dipolar interactions in LDHs. (See SI 13) The numerical results for a wide window of interlayer distances and spin correlation sizes are summarized in Fig 5. One observes that the dipolar interactions vary very little with the interlayer distance for spin correlation sizes above $15 \mathrm{~nm}$. Only when these spin correlation size becomes really small the interlayer distance need to be taken into account. The first qualitative conclusion is the fact that the factor governing dipolar interaction between magnetic layers is the layer size or, for more realistic multidomain layers, the domain size; only for tiny domain sizes (or high cation disorder) the interlayer distance do play a dominant role. ${ }^{44}$ The second main conclusion is that, for single-domain layers, increasing distances cannot change the sign of the dipolar magnetic coupling; this possibly changes for multi-domain layers. These results suggest that highly disordered layers showing cation clustering will be significantly affected by dipolar interactions. Furthermore, submicrometric LDH particles like those typically obtained through coprecipitation routes or using reverse microemulsion approaches will be more susceptible to suffer a stronger dipolar influence. Moreover, the introduction of two magnetic cations within the layers (e.g. NiFe or NiCo-LDHs) will lead to competing intralayer magnetic interactions and cation clustering as previously reported by our group, ${ }^{16}$ resulting in a more complex behavior in which, presumably, dipolar interactions will play a key role. 


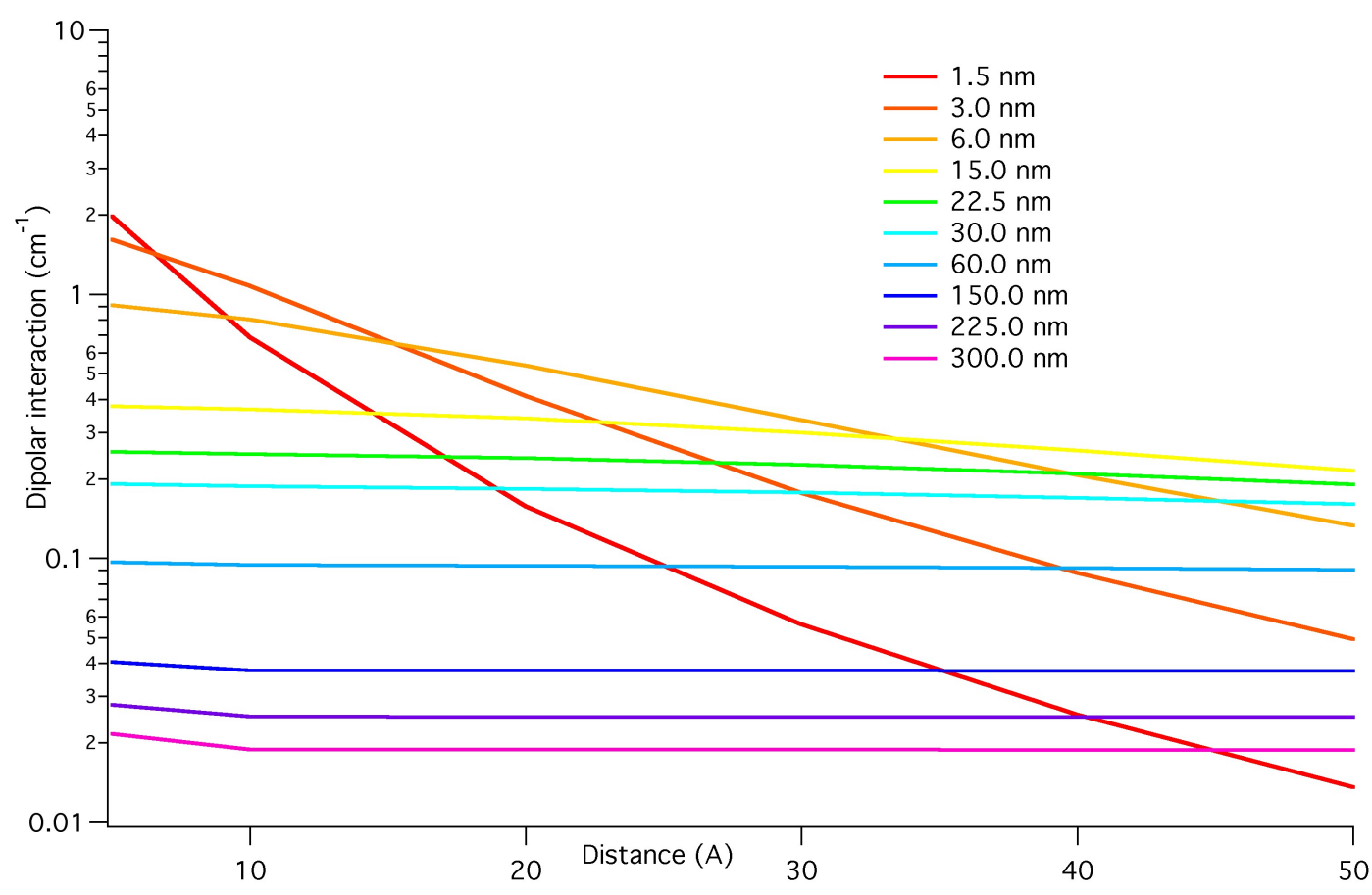

Fig. 5. Dipolar interaction variation with the interlayer distance and the spin correlation size on each layer.

Finally we have also performed AC dynamic susceptibility measurements in the presence of an applied field of 3.95 Oe in the $1-10000 \mathrm{~Hz}$ frequency range. The results confirm a cooperative magnetic behavior in all the samples, as seen in Fig.6A, SI 6 and SI 7. Both the in-phase $\left(X^{\prime}{ }_{M}\right)$ and the out-of-phase signals $\left(X^{\prime}{ }_{M}\right)$ displays defined peaks at low temperatures exhibiting frequency-dependence. From the out-of-phase signal, one can deduce the temperature for the onset for the spontaneous magnetization $\left(T_{M}\right)$, defined as the point where $x{ }_{\mathrm{M}} \neq 0$. All the values fall in the 4.8-5.4 K range, as expected for this sort of CoAl-LDH. This small variation in $\mathrm{T}_{M}$ indicates that the dominant factor controlling the cooperative behavior is not the interlayer distance. For the CoAl-ODS $\mathrm{T}_{\mathrm{M}} \approx 4.8 \mathrm{~K}$, which is not the highest value $\left(\mathrm{T}_{\mathrm{M}} \approx 5.4 \mathrm{~K}\right.$ in CoAl-ES). The frequency-dependence in this sort of materials is related with its intrinsically disordered nature and glassy behavior. ${ }^{45-47}$ We have confirmed the spin-glass nature of these compounds by extracting the frequency-shift parameter (also known as the Mydosh parameter $\phi$ ) from the out-of-phase signal. ${ }^{48}$

$$
\begin{gathered}
u \\
T_{\text {max }} \Delta \dot{i} \\
\Phi=\Delta T_{\text {max }} / \dot{i}
\end{gathered}
$$


We have found $\phi<0.1$ for all the samples, in good agreement with that found for related hybrid CoAl-LDHs and spin glass-like materials previously reported. ${ }^{10,43,48,49}$

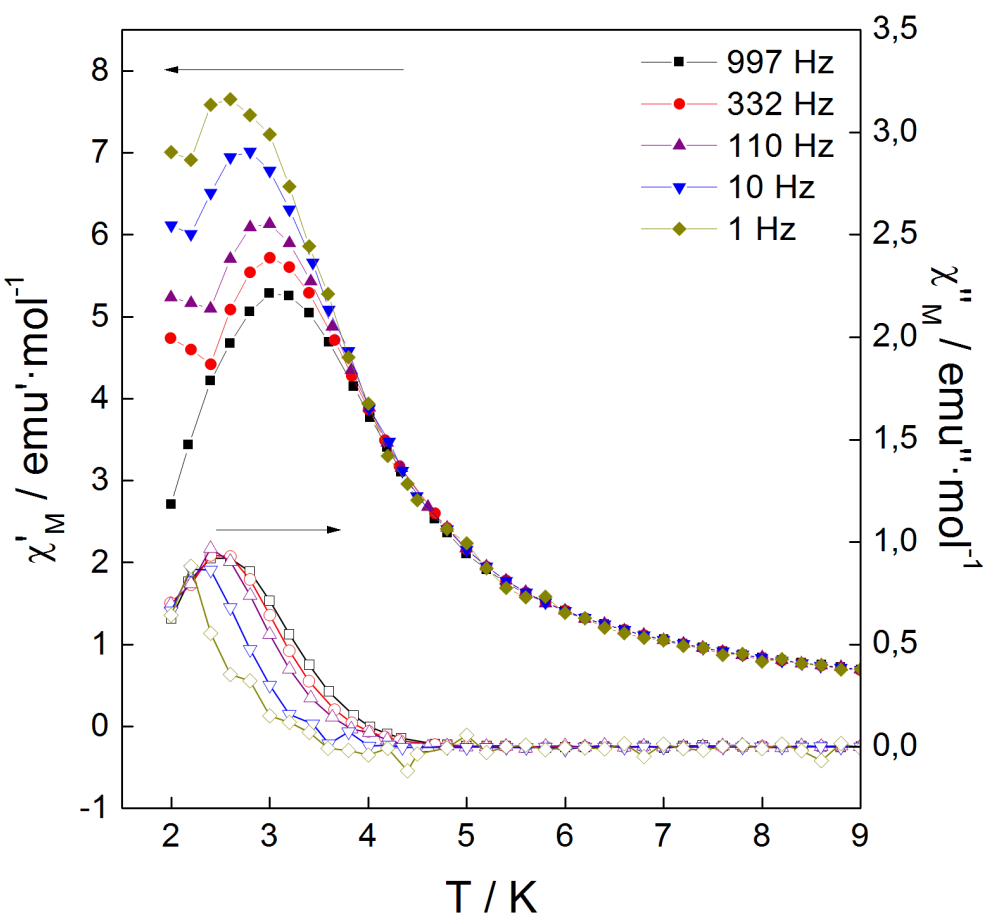

Fig. 6. CoAl-ODS sample: Thermal dependence of the $\chi$ ’M (in-phase) and $\chi$ ”M (out-ofphase) signals at 1, 10, 110, 332 and $997 \mathrm{~Hz}$.

\section{Conclusions}

A family of highly crystalline, hexagonal shaped hybrid CoAl-LDH with increasing basal spacing up to $34 \AA$ has been synthesized, in order to investigate the role exerted by the dipolar interactions in their overall magnetic behavior. The successful anion exchange reactions of the different surfactants in the interlayer space of the LDH system has been demonstrated by means of XRPD, FTIR or TGA analysis. The hexagonal morphology and platelet sizes are kept in every member of the CoAl-LDH family. A combined experimental and theoretical study has been developed showing a marked different behavior compared to homometallic layered hydroxides. Conversely to what is expected from a naive point of view, the study revealed that the major factor governing the dipolar interactions between magnetic layers is the spin correlation size, or, in the limit case, the lateral dimension of the layer, rather than the interlayer distance. This explains why the cooperative behavior observed in these materials is largely 
independent on the interlayer distance and suggests a strong influence of the cation ordering and particle dimensions on the magnetic behaviour. In the future we will study these effects, in particular the influence of the size effect on the magnetic properties of these layered materials, to check if the expected predictions are fulfilled. 


\section{References}

1P. Gómez-Romero and C. Sanchez, Eds., Functional hybrid materials, Wiley-VCH, Weinheim, 2004.

2E. Coronado, J. R. Galán-Mascarós, C. J. Gómez-García and V. Laukhin, Nature, 2000, 408, 447-449.

3E. Coronado and P. Day, Chem. Rev., 2004, 104, 5419-5448.

4E. Coronado and J. R. Galán-Mascarós, J. Mater. Chem., 2005, 15, 66-74.

5M. Clemente-León, E. Coronado, C. Martí-Gastaldo and F. M. Romero, Chem. Soc. Rev., 2011, 40, 473.

6E. Coronado and G. Mínguez Espallargas, Chem. Soc. Rev., 2013, 42, 1525.

7V. Rives, Layered Double Hydroxides: Present and Future, Nova Publishers, 2001.

8G. Abellán, C. Martí-Gastaldo, A. Ribera and E. Coronado, Acc. Chem. Res., 2015, 48, 1601-1611.

9Q. Wang and D. O’Hare, Chem. Rev., 2012, 112, 4124-4155.

10 G. Abellán, E. Coronado, C. Martí-Gastaldo, A. Ribera, J. L. Jordá and H. García, Adv. Mater., 2014, 26, 4156-4162.

11 G. Abellán, J. L. Jordá, P. Atienzar, M. Varela, M. Jaafar, J. Gómez-Herrero, F.

Zamora, A. Ribera, H. García and E. Coronado, Chem Sci, 2015, 6, 1949-1958.

12 G. Abellán, C. Martí-Gastaldo, A. Ribera and E. Coronado, Acc. Chem. Res., 2015, 48, 1601-1611.

13 C. Zhang, T. Tsuboi, H. Namba, Y. Einaga and T. Yamamoto, Dalton Trans, 2016, 45, 13324-13331.

14 E. Coronado, J. R. Galán-Mascarós, C. Martí-Gastaldo, A. Ribera, E. Palacios, M. Castro and R. Burriel, Inorg. Chem., 2008, 47, 9103-9110.

15 Z. Liu, R. Ma, M. Osada, N. Iyi, Y. Ebina, K. Takada and T. Sasaki, J. Am. Chem. Soc., 2006, 128, 4872-4880.

16 G. Abellán, E. Coronado, C. Martí-Gastaldo, J. Waerenborgh and A. Ribera, Inorg. Chem., 2013, 52, 10147-10157.

17 A. A. A. Ahmed, Z. A. Talib and M. Z. Hussein, Mater. Res. Bull., 2015, 62, $122-131$.

18 T. Yang, D. Xia, G. Chen and Y. Chen, Mater. Chem. Phys., 2009, 114, 69-72.

19 J. W. Lee, W. C. Choi and J.-D. Kim, CrystEngComm, 2010, 12, 3249.

20 H. Y. Yin, L. Wang, Y. F. Sun, D. J. Shi and X. X. Wang, Adv. Mater. Res., 2011, 233-235, 2091-2097.

21 N. A. Mazlan, N. Osman, A. M. Md Jani and M. H. Yaakob, J. Sol-Gel Sci. Technol., 2016, 78, 50-59.

22 C. J. Wang, Y. A. Wu, R. M. J. Jacobs, J. H. Warner, G. R. Williams and D.

O’Hare, Chem. Mater., 2011, 23, 171-180.

23 R. B. Viana, A. B. F. da Silva and A. S. Pimentel, Adv. Phys. Chem., 2012, 2012, $1-14$.

24 M. Meyn, K. Beneke and G. Lagaly, Inorg. Chem., 1993, 32, 1209-1215.

25 J. Zhang, Y. F. Xu, G. Qian, Z. P. Xu, C. Chen and Q. Liu, J. Phys. Chem. C, 2010, 114, 10768-10774.

26 J. A. Carrasco, J. Romero, M. Varela, F. Hauke, G. Abellán, A. Hirsch and E. Coronado, Inorg Chem Front, 2016, 3, 478-487.

27 J. Zhang, Z. Yang, J. Qiu and H.-W. Lee, J Mater Chem A, 2016, 4, 5802-5809.

28 G. Abellán, J. A. Carrasco, E. Coronado, J. Romero and M. Varela, J. Mater. Chem. C, 2014, 2, 3723-3731.

29 G. Abellán, E. Coronado, C. Martí-Gastaldo, E. Pinilla-Cienfuegos and A. Ribera, J. Mater. Chem., 2010, 20, 7451-7455. 
30 K. Okamoto, N. Iyi and T. Sasaki, Appl. Clay Sci., 2007, 37, $23-31$.

31 M. Ogawa and M. Hiramine, Cryst. Growth Des., , DOI:10.1021/cg401684u.

32 V. Laget, C. Hornick, P. Rabu and M. Drillon, J. Mater. Chem., 1999, 9, 169174.

33 P. Rabu and M. Drillon, Adv. Eng. Mater., 2003, 5, 189-210.

34 P. Rabu, E. Delahaye and G. Rogez, Nanotechnol. Rev., 2015, 4, 557-580.

35 G. Rogez, C. Massobrio, P. Rabu and M. Drillon, Chem. Soc. Rev., 2011, 40, 1031.

36 J. D. Rall and M. S. Seehra, J. Phys. Condens. Matter, 2012, 24, 76002.

37 M. S. Seehra and V. Singh, J. Phys. Condens. Matter, 2013, 25, 356001.

38 G. Abellán, F. Busolo, E. Coronado, C. Martí-Gastaldo and A. Ribera, J. Phys. Chem. C, 2012, 116, 15756-15764.

39 G. Layrac, D. Tichit, J. Larionova, Y. Guari and C. Guérin, J. Phys. Chem. C, 2011, 115, 3263-3271.

40 G. Abellán, E. Coronado, C. J. Gómez-García, C. Martí-Gastaldo and A. Ribera, Polyhedron, 2013, 52, 216-221.

41 G. Abellán, J. A. Carrasco and E. Coronado, Inorg. Chem., 2013, 52, 7828-7830.

42 Y. Kobayashi, X. Ke, H. Hata, P. Schiffer and T. E. Mallouk, Chem. Mater., 2008, 20, 2374-2381.

43 C. J. Wang, Y. A. Wu, R. M. J. Jacobs, J. H. Warner, G. R. Williams and D. O’Hare, Chem. Mater., 2011, 23, 171-180.

44 J. Pérez-Ramírez, A. Ribera, F. Kapteijn, E. Coronado and C. J. Gómez-García, J. Mater. Chem., 2002, 12, 2370-2375.

45 G. Abellán, E. Coronado, C. Martí-Gastaldo, J. Waerenborgh and A. Ribera, Inorg. Chem., 2013, 52, 10147-10157.

$46 \quad$ S. Cadars, G. Layrac, C. Gérardin, M. Deschamps, J. R. Yates, D. Tichit and D. Massiot, Chem. Mater., 2011, 23, 2821-2831.

47 S. S. C. Pushparaj, C. Forano, V. Prevot, A. S. Lipton, G. J. Rees, J. V. Hanna and U. G. Nielsen, J. Phys. Chem. C, 2015, 119, 27695-27707.

48 J. A. Mydosh, Spin glasses: an experimental introduction, Taylor \& Francis, London ; Washington, DC, 1993.

49 N. Tanaka, A. Okazawa, A. Sugahara and N. Kojima, Bull. Chem. Soc. Jpn., 2015, 88, 1150-1155. 